\title{
Decisiones en evaluación: ambientes virtuales de posgrado, un estudio ex post-facto.
}

\section{Decisions in evaluation: virtual postgraduate environments, ex post-facto study.}

\author{
Viviana Rocio Rodriguez Aramendiz \\ Instituto Tecnológico y de Estudios Superiores de Monterrey, Monterrey, México \\ vivianarodrigueza@gmail.com \\ Katherina Edith Gallardo Córdova \\ katherina.gallardo@itesm.mx \\ Instituto Tecnológico y de Estudios Superiores de Monterrey, Monterrey, México
}

\section{Resumen}

Esta investigación se realizó en torno a la toma de decisiones relacionada con la evaluación del aprendizaje. El análisis abarcó tanto el aspecto instruccional como el de retroalimentación a actividades evaluables. Se tomaron dos materias o cursos ofrecidos en programas en línea a nivel posgrado. El marco conceptual de referencia fue la Nueva Taxonomía de Marzano y Kendall (2007) el cual permitió emitir una serie de juicios sobre el diseño y la retroalimentación. Se optó por un diseño ex post-facto para el análisis de tareas, actividades asignadas y los objetivos o competencias para el cumplimiento de las metas generales.

Palabras clave: evaluación; taxonomía; objetivo; competencia.

\begin{abstract}
This research was conducted to analyze the competence of decisions making for learning evaluation matters. The analysis covered both the instructional aspect and the feedback given to assessable activities. Two subjects or courses offered on the online modality for graduate level were analyzed. The New Taxonomy (Marzano and Kendall, 2007) was used as a conceptual framework that permitted the emission of a series of judgments on design and feedback. An ex post-facto study permitted the analysis of tasks, assigned activities and objectives or competencies to fulfill the overall educational goals.
\end{abstract}

Keywords: evaluation; taxonomy; objective; competence. 


\section{Introducción}

La educación busca dar respuesta a los cambios culturales y económicos que se suscitan en los diferentes ámbitos de desarrollo, insertando en sus procesos alternativas para lograr una interacción respaldada por TIC. Por su parte, estas se han convertido en una herramienta indispensable en el diseño y operación de los ámbitos formativos, en especial en la educación superior.

Las TIC se convierten en recursos flexibles y de fácil acceso que permiten el diseño de ambientes de aprendizaje. Así, hoy en día es más factible conducir procesos dinámicos de enseñanza- aprendizaje sin que la localización de sus participantes sea un factor que impida dicho dinamismo. Según Martínez Uribe (2008) las nuevas tecnologías apoyan la difusión de información de modo eficiente y permiten realizar un diseño diferencial entre lo presencial y en línea, además de dotar a los participantes de herramientas para la comunicación y el aprendizaje colaborativo. De hecho, en los últimos años, los ambientes de enseñanza - aprendizaje a distancia han despertado un alto interés, abriendo posibilidades para el desarrollo de nuevos modelos pedagógicos. Solo en la última década, según el Consorcio de Educación a Distancia (2014), el incremento de alumnos universitarios que toman un curso en la modalidad a distancia es de $3.7 \%$. Además, el $70.8 \%$ de líderes académicos en diversas instituciones reconoce la importancia de la modalidad a distancia, la cual es indispensable implementarla en los siguientes años para mantener la oferta académica atractiva para su público meta.

La educación a distancia antes mencionada logra su oferta a través de una serie de procesos propios del diseño instruccional, los cuales se logran a partir de la convergencia de las teorías pedagógicas orientada por las metas, el contexto, la modalidad, el nivel educativo y la incorporación de la tecnología como agente dinamizador del proceso educativo (Córica et. al., 2010; Londoño Giraldo, 2011; Molenda, 1997). La evaluación del aprendizaje en el marco del diseño instruccional se concibe y realiza frecuentemente a priori, se vuelve indispensable para asegurar que la entrega de los contenidos y las actividades serán suficientes para poder estimar qué tanto los estudiantes han alcanzado las metas de aprendizaje.

Un posible problema que se puede suscitar es que el experto quien diseña cursos en la modalidad a distancia no considere del todo la naturaleza de los objetivos o competencias y la forma de vincularlas con el modelo educativo, afectando los procesos de evaluación del aprendizaje (Gros Salvat, 2012). Por lo anterior, es importante indagar sobre cómo los diseñadores expertos de cursos o materias en las diferentes disciplinas aplican la teoría y modelo educativo de la cual parten,

Decisiones en evaluación: ambientes virtuales de posgrado, un estudio ex postfacto. Viviana Rocio Rodriguez Aramendiz y Katherina Edith Gallardo Córdova 
para que los estudiantes logren el dominio de conceptos y el desarrollo de diferentes habilidades en el marco de sus materias. Así, dichos expertos integran diferentes teorías educativas y de evaluación del aprendizaje que promueven la adquisición de habilidades para solucionar problemas entre otras competencias, a través del diseño instruccional de sus cursos (Londoño Giraldo, 2011).

Por lo anterior, surgen los siguientes interrogantes de investigación: ¿Cómo valorar las decisiones desde el diseño instruccional y luego en la operacionalización de la evaluación del aprendizaje en ambientes virtuales? ¿Qué prácticas se están llevando a cabo que permitan valorar si las decisiones tomadas en cuanto a la evaluación del aprendizaje llevan a cumplir con los objetivos o competencias estimadas?.

\section{Marco Teórico}

\section{Evaluación del aprendizaje}

La evaluación es indispensable para la calidad educativa, pues permite entender los procesos de enseñanza-aprendizaje (Gallardo Córdova, 2013). La evaluación ha sufrido diferentes cambios y transformaciones conceptuales a lo largo de la historia. En la edad media se aplicaron los exámenes públicos orales con un carácter más formal. A finales del siglo XIX se despierta el interés por la medición científica donde la primera investigación evaluativa recibe influencias de otras disciplinas pedagógicas como la pedagogía experimental y diferencial.

La evaluación del aprendizaje recibe cierta influencia de otras disciplinas durante el siglo XX, Madaus, Scriven, Stufflebeam (citados en Escudero Escorza, 2003) se toma como punto de partida la obra Tyler, donde se destacan las diferentes generaciones a largo de su evolución como: el enfoque paradigmático constructivista, la medición, la descripción y el juicio o valoración. Se dan los primeros inicios y diálogos de evaluación durante la década de 1920 y 1930 denominada la primera generación de la medida donde surgen los denominados test estandarizados para medir destrezas, con la intención de determinar la inteligencia.

Seguido del surgimiento de test estandarizados, la evaluación se orienta hacia los individuos en la década de 1970, donde se consolidan las próximas investigaciones en evaluación. Surge la diversificación de fuentes del proceso y juicios evaluativos, estableciendo criterios de validez y confianza donde se integran los actores del proceso educativo (Escudero Escorza, 2003). La Figura 1 consolida estos momentos históricos de la evolución de la evaluación.

Decisiones en evaluación: ambientes virtuales de posgrado, un estudio ex postfacto. Viviana Rocio Rodriguez Aramendiz y Katherina Edith Gallardo Córdova 


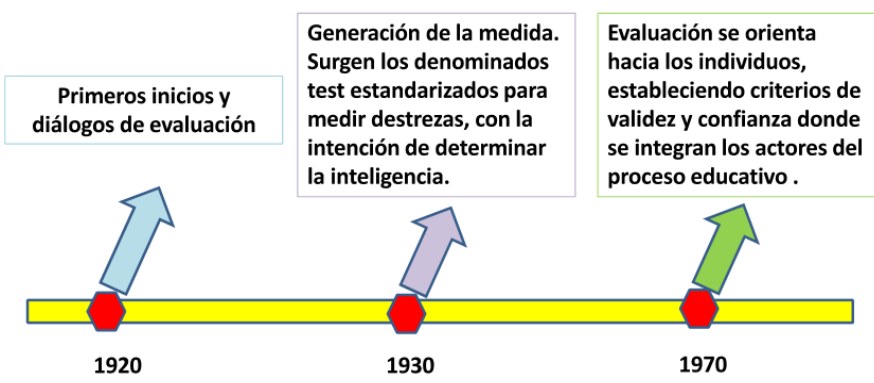

Figura 1. Evolución de la evaluación durante el siglo XX (basado en la obra de Tyler).

Según los estudios realizados a lo largo de estos años, la evaluación debe contar con las siguientes características: ser integral, sistemática, participativa y continúa. Se le considera integral pues abarca todos y cada uno de los elementos y actores del proceso educativo, el contexto socio-económico, cultural y las dimensiones del individuo que afectan el aprendizaje; sistemática pues se debe planificar, utilizar instrumentos confiables, donde se formulan el aprendizaje a evaluar. Su rasgo de participativa hace que el proceso contemple la intervención de diferentes actores a fin de posibilitar el mejoramiento de lo aprendido mediante la autoevaluación, coevaluación y heteroevaluación. Finalmente, es considerada continua ya que se realiza en diferentes momentos dando resultados durante todo el proceso (Casanova Rodríguez, 1998). Todos estos aspectos mencionados determinan la evaluación como una actividad previa conducente a elevar la calidad educativa (De La Garza Vizcaya, 2004).

Uno de los tipos de evaluación que se ha definido y evolucionado en los últimos años es la evaluación formativa (Jané, 2005). Este tipo de evaluación está enfocada en verificar el progreso del desempeño de los estudiantes en la dirección que marcan las metas de aprendizaje planteados a priori. Así, su principal propósito es permitir la toma de decisiones frente a las alternativas de acción y dirección a medida que el proceso de enseñanza-aprendizaje avanza, retroalimentar con información obtenida de los exámenes para finalmente dirigir el aprendizaje hacia la eficacia y regular el ritmo del aprendizaje. Por lo anterior, se sabe que el proceso de evaluación formativa se complementa cuando se lleva a cabo la retroalimentación que reúne información con base en la cual se pueden realizar inferencias frente al nivel de dominio logrado por el estudiante, las capacidades desarrolladas, los contenidos, su potencial y respuesta en situaciones reales que permitan aplicar lo aprendido (Benítez Lima, 2010).

Decisiones en evaluación: ambientes virtuales de posgrado, un estudio ex postfacto. Viviana Rocio Rodriguez Aramendiz y Katherina Edith Gallardo Córdova 


\section{El papel de la evaluación en el proceso de enseñanza- aprendizaje}

La evaluación del aprendizaje se considera como un proceso continuo que coadyuva a determinar el grado en el que los estudiantes logran alcanzar los objetivos. Sin duda, es un elemento indispensable del currículo, pues permite obtener resultados de aprendizaje además de los estándares de calidad logrados en el proceso enseñanza- aprendizaje.

Existen diferentes funciones del proceso evaluativo. Una de ellas es la función pedagógica que cumple con la introspección en el proceso enseñanza- aprendizaje, a fin de buscar una mejora. Esta función permite verificar el alcance de las competencias y/o adquisición de conocimientos por parte de los estudiantes. Además, superar y reforzar dificultades, motivar el alcance de nuevos aprendizajes y dar seguimiento a la aplicación de medidas para la implementación de mejoras, buscando la regulación y autonomía del aprendizaje. Esta función también integra el análisis de resultados obtenidos desarrollados para la identificación de fortalezas y debilidades del proceso educativo (Alkharusi, 2008; Beauvais, s/f; De Vizenci y De Angelis, 2008).

Otra función que debe considerarse dentro de las aportaciones fundamentales de la evaluación del aprendizaje es la de comunicación hacia la sociedad. Es a través de la evaluación que se pueden otorgar reconocimientos y acreditaciones de los diferentes niveles del sistema educativo, certificando el logro de aprendizajes en un periodo determinado. Así, la evaluación del aprendizaje ejerce influencia en los contenidos, la planeación y didáctica para la realización de ajustes frente al contexto, perfil de los estudiantes y el proceso en sí mismo, convirtiéndose en un mecanismo para verificar el alcance de las metas educativas (Stugis y Patrick, 2010).

\section{Aspectos básicos del diseño instruccional}

El diseño instruccional ese basa en la creación y diseño de módulos, fundamentada en la tecnología educativa, entendida como la aplicación de la tecnología para la elaboración de recursos de aprendizaje como: guiones, planes, proyectos llevados a cabo bajo procedimientos estandarizados (Londoño Giraldo, 2011). El diseño instruccional se puede aplicar en diferentes modalidades tal como lo es la educación a abierta y a distancia. En los modelos de diseño instruccional se tienen en cuenta pasos con la intención de producir y generar la optimización de la enseñanza y del aprendizaje, el camino o guía que todo educador debe trazar al pretender dirigir un curso, independiente de su modalidad. Martínez Rodríguez (2009) plantea que el trabajo de diseño instruccional debe ser ecléctico y realizado por un equipo experto e interdisciplinario, compuesto por el diseñador instruccional quien conoce y profundiza en las fortalezas y debilidades de las

Decisiones en evaluación: ambientes virtuales de posgrado, un estudio ex postfacto. Viviana Rocio Rodriguez Aramendiz y Katherina Edith Gallardo Córdova 
teorías de aprendizaje para optimizar el uso pertinente de las estrategias y enriquecer el trabajo a desarrollar. Así, la tecnología se convierte en un medio de aprendizaje e intercambio de información sin límites de tiempo y distancia.

\section{Diseño instruccional para cursos que se ofrecen en la modalidad en línea}

El diseño instruccional ha sufrido cambios a lo largo de la historia. Se resaltan cuatro generaciones que sustentan su evolución en diferentes teorías de aprendizaje (Benítez Lima, 2010). En 1960 se establecieron modelos fundamentados en el conductismo: sistemáticos, lineales y prescriptivos; relacionados con las destrezas, conocimientos, objetivos de aprendizaje, observables y medibles. En 1970 se fundamenta la teoría de sistemas, especialmente abiertos. En 1980 se fundamenta la teoría cognitiva preocupada por procesos mentales y su comprensión, entre ellos, la formación de conceptos y el procesamiento de información, la solución de problemas. Finalmente, en 1990, se fundamentan las teorías constructivistas y de sistemas, centradas en el proceso de aprendizaje dejando de lado los contenidos y la creatividad del estudiante.

Teniendo en cuenta su evolución, podría afirmarse que un buen modelo para el diseño instruccional se logra a partir de la convergencia del contexto, las teorías pedagógicas orientadas por las metas, el nivel educativo, la modalidad, y la incorporación de la tecnología como agente dinamizador del proceso educativo. El objetivo principal de los modelos se basa en la presentación de diferentes actividades de evaluación, contenidos y aprendizaje. Se agrupan según sus propósitos y orígenes en tecnología y desarrollo de procesos genéricos y conceptos de diseños de aprendizaje o teorías.

Desde una perspectiva más operativa, en la educación a distancia generalmente se opta por realizar los procesos de diseño instruccional a priori, pues al momento de ser entregado al estudiante el modulo, curso o materia debe estar integrado en su totalidad junto con las actividades de aprendizaje, con la finalidad de dar un marco de referencia y seguridad a los estudiantes sobre qué aprenderá y cómo demostrará su desempeño. Este factor detona una de los interrogantes que se tratan de estudiar en esta investigación.

\section{Toma de decisiones en la evaluación del aprendizaje: apoyo en una taxonomía de aprendizaje}

La toma de decisiones es esencial en las prácticas de evaluación formativa, pues se deriva de la demanda de evidencias de aprendizaje, las cuales deben permitir la inferencia de lo que el estudiante reconoce, analiza y/o utiliza el conocimiento, teniendo en cuenta los contenidos estudiados. Las decisiones sobre qué evidencias

Decisiones en evaluación: ambientes virtuales de posgrado, un estudio ex postfacto. Viviana Rocio Rodriguez Aramendiz y Katherina Edith Gallardo Córdova 
de aprendizaje solicitar deben sostenerse en conocimientos relacionados con los procesos de pensamiento y la naturaleza de la disciplina en estudio e ir más allá de conocer las técnicas para elaborar dichos productos (Gallardo Córdova, 2013).

Una alternativa para tomar decisiones en evaluación puede apoyarse en el uso de la Nueva taxonomía propuesta por R Marzano y J. Kendall basada en la propuesta de Benjamín Bloom de 1956. Esta propuesta taxonómica parte de diferentes estudios de los procesos de pensamiento y dominios de conocimiento. Constituye una alternativa para identificar dónde se está y a dónde se quiere llegar en el proceso de enseñanza aprendizaje. También recibe el nombre de la Nueva taxonomía de objetivos educativos (Marzano y Kendall, 2007).

Esta taxonomía está conformada por dos dimensiones, niveles de procesamiento y dominios de conocimiento. Los niveles de procesamiento conforman tres sistemas (interno, metacognitivo y cognitivo), en cuanto a los dominios de conocimiento se clasifica en tres (procedimientos mentales y psicomotores e información). La Nueva taxonomía establece cuatro niveles de procesamiento, que van desde la Recuperación, la Comprensión el Análisis y la Utilización de conocimiento.

Sobre los dominios de conocimiento, estos son: la Información como un conjunto de datos que permiten la descripción de manera sistematizada, clasificada en: vocabulario, hechos, secuencia, generalizaciones y principios. Por su parte, los procedimientos mentales corresponden al manejo de información procedimental para ejecutar acciones, clasificando el aprendizaje de los procedimientos mentales en reglas simples, algoritmos, tácticas y macroprocedimientos. También contempla procedimientos psicomotores relacionados con la coordinación de movimientos con velocidad, fuerza y firmeza (Marzano y Kendall, 2007).

Cabe destacar que este marco taxonómico ha sido utilizando en diferentes investigaciones educativas relacionadas con disciplinas como medicina, ingeniería, química, educación y economía etc. (Desai y Johnson, 2014; Dubas y Toledo, 2016; Law y Kaufhold, 2009; Shelton y Brown, 2010). En una de estas recientes investigaciones (Dubas y Toledo, 2016) se argumenta la necesidad de los profesores de fomentar de forma intencionada las habilidades de pensamiento de orden superior en los estudiantes, específicamente en el área de economía. El interés de propiciar estos niveles de procesamiento mental subyace en que los conocimientos se hacen más duraderos y flexibles, siendo posible realizar procesos de transferencia.

La nueva taxonomía fue utilizada en el diseño de cursos como en la emisión de los resultados del aprendizaje del estudiante. Los resultados giran alrededor de dos aspectos: el primero sobre el diseño y la cantidad de actividades que propician procesos de pensamiento tales como la comprensión y el análisis. Los diseñadores declaran no haber considerado en anteriores diseños suficientes actividades que proporcionen un andamiaje robusto para propiciar estos procesos de pensamiento

Decisiones en evaluación: ambientes virtuales de posgrado, un estudio ex postfacto. Viviana Rocio Rodriguez Aramendiz y Katherina Edith Gallardo Córdova 
en los estudiantes. Un segundo aspecto que se resalta en los resultados, es el hecho de haber podido entregar a los estudiantes elementos de retroalimentación más enfocados a los niveles de pensamiento al que se quería llegar.

\section{Método}

La metodología permite estudiar los hechos ocurridos sin manipulación, es decir se estudian en función de las características que posean. Por su parte el investigador no tiene control de las variables y sus efectos ya sucedieron. Las variables no son controladas justificando la investigación mediante la descripción de los procesos evaluativos de cada curso virtual y su eficacia y eficiencia además, de analizar los datos cualitativos y cuantitativos permitiendo según Valenzuela Gonzáles y Flores Fahara (2012) la interpretación de información previamente registrada. Se considera además la estrategia de investigación retrospectiva, donde el proceso casual ya ha ocurrido, buscando las causas que los han ocasionado para reconstruir los hechos.

Para conducir este estudio sobre toma de decisiones en evaluación, en el marco de cursos en la modalidad a distancia, se optó por el uso de Métodos mixtos. Según Creswell y Plano (2007) y Valenzuela González y Flores Fahara (2012) este método tiene como objetivo la mezcla, el análisis y colección de datos cualitativos y cuantitativos que están enlazados con la pregunta de investigación y buscan responderla a partir de una variedad de datos, con la certeza de que esta metodología pueda ayudar a entender los fenómenos de una manera más profunda.

La naturaleza del estudio demandó utilizar un enfoque ex post facto dado que se analizaron cursos en línea y a distancia que iniciaron y terminaron en un tiempo anterior a la realización de la indagación. El enfoque ex post facto de tipo retrospectivo simple se caracteriza, según Hernández Sampieri, Fernández Collado, Baptista Lucio (2006), en que después de los hechos ocurridos, no se manipulan los datos ni se asignan sujetos de estudio de manera aleatoria. De hecho, estos se eligen en función de las características que posean. Por su parte, el investigador no tiene control sobre las variables. Los efectos ya sucedieron y se encuentran evidenciados y/o registrados.

El escenario seleccionado para esta investigación fue el área de programas de posgrado en educación y administración de una universidad al noreste de México, la cual sostiene una oferta de educación a distancia desde hace más de dos décadas. Se solicitó consentimiento de los directores de programa, obteniendo acceso a los cursos "Liderazgo y comportamiento organizacional" denominado de ahora en adelante como el Curso A y "Evaluación de instituciones educativas" denominado el Curso B.

Decisiones en evaluación: ambientes virtuales de posgrado, un estudio ex postfacto. Viviana Rocio Rodriguez Aramendiz y Katherina Edith Gallardo Córdova 


\section{Objetivos}

Reconocer las decisiones tomadas frente a las prácticas evaluativas en los ambientes virtuales de aprendizaje.

Identificar la efectividad de las decisiones en el proceso evaluativo para alcanzar los objetivos llevado a cabo en los programas modalidad en línea.

Determinar la relación de los procesos evaluativos con los niveles cognitivos declarados en los objetivos o competencias de aprendizaje.

\section{Población y Muestra}

La investigación se llevó a cabo en un ambiente de aprendizaje virtual analizando los sistemas de evaluación de dos cursos de posgrado en línea ya finalizados. Estos programas son ofrecidos a profesionales en el área educativa teniendo en cuenta la inclinación académica del investigador.

En los dos cursos participaron un total de 230 estudiantes y 6 profesores divididos de la siguiente forma: en "Curso A" con 163 estudiantes y 3 profesores y en "Curso B" con una cantidad de 67 estudiantes y 3 profesores, pertenecientes al área que atiende la oferta de educación a distancia.

\section{Instrumentos}

Los instrumentos según las obras de Marzano y Kendall (2007), Mc.Vay. (2002) y Morgan y O'Reilly (2002) constituyen en realizar un vaciado de los cursos estudiados mediante dos hojas excel compartidas con información básica de cada curso, teniendo en cuenta los siguientes aspectos: datos generales, datos sobre procesos de evaluación, objetivos y competencias declaradas, dominios y niveles de procesamiento y análisis profundo de las actividades ponderadas.

\section{Procedimiento de recogida y análisis de datos}

En cuanto al procedimiento del estudio se analizó cada curso en tres momentos, teniendo en cuenta los aspectos mencionados:

1. Indagación global en los cursos seleccionados teniendo en cuenta elementos generales de los procesos de evaluación. Este primer análisis permitió tener una visión holística de cada curso en cuanto a los objetivos y estrategias para lograr alcanzar las metas formativas.

2. Indagación sobre alcances de los objetivos/competencias a través de las actividades seleccionadas tomando la Nueva taxonomía como marco de referencia (Marzano y Kendall, 2007). Este segundo análisis permitió entender la

Decisiones en evaluación: ambientes virtuales de posgrado, un estudio ex postfacto. Viviana Rocio Rodriguez Aramendiz y Katherina Edith Gallardo Córdova 
finalidad de cada actividad en relación con las demás, para trabajar en cada etapa del proceso formativo.

3. Análisis de los datos en materia de retroalimentaciones emitido a las actividades ponderadas. Este tercer análisis reflejó qué tanto los comentarios sobre fortalezas y debilidades percibidas en las evidencias de trabajo se encausaron para ayudar a los estudiantes en el logro de las metas de aprendizaje. Se recuperaron tareas o productos retroalimentados con calificaciones entre $70 \mathrm{y}$ 85 , por considerarse productos que podrían tener más comentarios sobre los logros que otros trabajos. Se analizaron un total de 20 tareas por curso.

\section{Resultados}

La investigación se orientó en el análisis de la evaluación que según Martínez Rizo (2013) es un proceso formativo que muestra el avance en el desarrollo de competencias y conocimientos de los alumnos y la toma de decisiones en dos programas en línea, basados en la nueva taxonomía.

El análisis y recolección de datos se presentó según el orden en que se analizaron los cursos: un primer momento donde se analizaron los procesos de evaluación; un segundo momento donde se revisaron los datos sobre alcances de los objetivos y competencias y finalmente, un tercer momento donde se analizan los datos obtenidos de las retroalimentaciones dadas a las actividades ponderadas en los cursos seleccionados

Momento 1: Indagación global en los cursos seleccionados sobre procesos de evaluación. Se inicia con el análisis de los componentes de evaluación del aprendizaje de cada uno de los cursos para determinar sus elementos y comprender la estructura del sistema de evaluación del aprendizaje. Se contabilizaron una serie de actividades involucradas con procesos de aprendizaje que tuvieran o no un valor o ponderación. A continuación se presenta la Tabla 1 que condensa estos elementos en el Curso A como en el B.

Decisiones en evaluación: ambientes virtuales de posgrado, un estudio ex postfacto. Viviana Rocio Rodriguez Aramendiz y Katherina Edith Gallardo Córdova 
Tabla 1.

Elementos que conformaron los sistemas de evaluación de los cursos $A$ y B

\begin{tabular}{lll}
\hline & Curso A & Curso B \\
\hline Número de unidades/módulos & 3 & 4 \\
Número de actividades individuales & 12 & 6 \\
Número de actividades grupales & 6 & 3 \\
Número de actividades automatizadas & 2 & 2 \\
Número de actividades no automatizadas (que requieren & 16 & 7 \\
retroalimentación y calificación por parte de un tutor) & & \\
Número de exámenes a presentar & 2 & 2 \\
Número de exámenes que tienen ponderación & 2 & 2 \\
Número de actividades que tiene ponderación o puntaje & 18 & 9 \\
\hline
\end{tabular}

Momento 2: Indagación sobre alcances de los objetivos/competencias a través de las actividades seleccionadas. Tanto el en curso A como en el B se realizó el análisis del objetivo, estructura y contenido de cada actividad ponderada, relacionando el nivel cognitivo exigido con el alcance de la competencia $u$ objetivos de aprendizaje a partir de las actividades reunidas alrededor de estos. A continuación, se presenta la Tabla 2 que conjunta un análisis sobre los alcances de las competencias u objetivos de ambos cursos y el nivel taxonómico al que aspira llegar.

Tabla 2.

Objetivos/competencias de los cursos A y B vistos desde la Nueva Taxonomía

\begin{tabular}{lll}
\hline Curso & Objetivos/Competencias & $\begin{array}{c}\text { Nivel de } \\
\text { procesamiento }\end{array}$ \\
\hline A & $\begin{array}{l}\text { Identificar cómo el comportamiento organizacional se ve afectado } \\
\text { por los cambios del entorno interno y externo a la empresa }\end{array}$ & Análisis \\
& $\begin{array}{l}\text { fundamental para el diseño de la gestión del capital humano y } \\
\text { que responda a estos imperativos. }\end{array}$ & \\
& $\begin{array}{l}\text { Reconocer los beneficios de elaborar proyectos relacionados con la } \\
\text { gestión del factor humano a través de equipos colaborativos } \\
\text { multidisciplinarios. }\end{array}$ & Comprensión \\
& $\begin{array}{l}\text { Aplicar el pensamiento crítico hacia la mejora de la optimización } \\
\text { del rol del líder de la organización como generador y animador de } \\
\text { los procesos humanos encaminados a incrementar el capital } \\
\text { humano de la organización. }\end{array}$ & \\
& &
\end{tabular}

Decisiones en evaluación: ambientes virtuales de posgrado, un estudio ex postfacto. Viviana Rocio Rodriguez Aramendiz y Katherina Edith Gallardo Córdova 
Emplear estrategias de liderazgo y cambio organizacional para optimizar la efectividad global de los procesos humanos.

Ser ejemplo digno a seguir por sus actuaciones ricas en valores y principios, basado en una ética que inspire confianza, transparencia y lealtad con sus trabajadores.

B

Aplicar estrategias de gestión de los recursos humanos, materiales y financieros de manera efectiva y eficiente para la planeación, ejecución y evaluación del quehacer académicoadministrativo.

Liderar proyectos que respondan a necesidades particulares de naturaleza académico-administrativas y que permitan resolver problemas y tomar decisiones
Utilización

conocimiento

Utilización

conocimiento

Comprensión

Utilización conocimiento

Reconocer y clasificar las competencias $\mathrm{u}$ objetivos de aprendizaje en el marco taxonómico sirvió como punto inicial de análisis para entender la naturaleza y seguimiento de las actividades ponderables. De esta forma podrían emerger una serie de juicios sobre la pertinencia de dichas actividades en función de las metas de aprendizaje a alcanzar. A continuación, se presenta la Tabla 3 que contiene los resultados del análisis sobre las actividades ponderadas por curso y el nivel de procesamiento en las que se clasificaron según el marco taxonómico.

Tabla 3.

Actividades y su relación con el nivel de procesamiento para el curso $A$ y $B$

$\begin{array}{llll}\text { Curso Actividad } & \text { Recuperación Comprensión } & \text { Análisis } & \begin{array}{l}\text { Utilización del } \\ \text { conocimiento }\end{array}\end{array}$

\begin{tabular}{|c|c|c|c|}
\hline \multirow[t]{8}{*}{ A } & 2.4 Resumen & $x$ & \\
\hline & 3.5 Resumen & $x$ & \\
\hline & 6.5 Resumen & $x$ & \\
\hline & $\begin{array}{ll}2.5 & \text { Foro } \\
\text { discusión } & \end{array}$ & & $X$ \\
\hline & $\begin{array}{ll}4.5 & \text { Foro } \\
\text { discusión } & \end{array}$ & & $X$ \\
\hline & $\begin{array}{ll}7.6 & \text { Foro } \\
\text { discusión } & \end{array}$ & & $X$ \\
\hline & $\begin{array}{l}8.7 \text { Discusión y } \\
\text { reporte }\end{array}$ & & $X$ \\
\hline & $\begin{array}{lr}10.7 \text { Foro } & \text { de } \\
\text { discusión } & y\end{array}$ & & $X$ \\
\hline
\end{tabular}

Decisiones en evaluación: ambientes virtuales de posgrado, un estudio ex postfacto. Viviana Rocio Rodriguez Aramendiz y Katherina Edith Gallardo Córdova 
B

reporte

2.5 Resumen
$\begin{aligned} & \text { 2.7 Ficha de } \\ & \text { datos }\end{aligned}$

3.7 Informe

proyecto

3.5 Proyecto

4.3 Proyecto

2.8 Comentario

X

3.6 Proyecto

En caso del curso A, se tuvo en cuenta al inicio del módulo, las actividades evaluables 2.4 3.5, y 6.5. Cada una de ellas permitió a los estudiantes trabajar en la apropiación de los contenidos básicos del tema a través de textos y videos, por lo cual se estimó que tanto la intención como tipo de producto (resumen) condujo a trabajar en el primer nivel cognitivo. Las actividades 2.5, 4.5, y 7.6 correspondieron a la ejecución de un proyecto grupal. Estas se planearon para fortalecer el diálogo en cada etapa, propiciando el intercambio de ideas fundamentadas, exigiendo llegar a la emisión de conclusiones. Este tipo de actividades promovieron procesos de análisis en los participantes pues requirieron asociar ideas, jerarquizar las más importantes y emitir opiniones sustentadas desde la teoría contrastándolas con la realidad, en diversas situaciones. Los foros llevaron a trabajar a los estudiantes en el nivel de análisis y utilización de conocimientos respectivamente.

Las actividades 8.7 y 10.7 se plantearon con la finalidad de abrir marcos de diálogo para analizar y reflexionar en el marco del Método de casos. Se clasificaron por lo tanto como actividades que involucran el análisis, la identificación de detalles importantes, la emisión de supuestos para encaminar el diálogo hacia una posible emisión de soluciones, sin que se llegue al proceso de implementación.

En el Curso B, la actividad 2.5 estuvo encaminada a iniciar con el proceso de apropiación de conceptos básicos de la disciplina a partir de lecturas sugeridas. La actividad 2.7 se planteó con la intención de realizar un proceso de reconocimiento de la institución educativa y sus indicadores claves, que sirvieran como puntos de referencia mínima requerida para posteriormente analizar, reflexionar e interpretar la calidad de los servicios diversos de la institución. Ambas actividades se ubican en el nivel de Reconocimiento pues su principal objetivo es el trabajo con ciertos detalles para iniciar las bases de conocimiento en la disciplina.

La documentación de indicadores claves que corresponden a las actividades 3.7, 3.5 y 4.3 cumplen con el objetivo documentar la información de la institución, además de responder y analizar unas preguntas concernientes a la misma, la actividad se desarrolló por fases. Esta información se requiere para dar soluciones específicas luego de analizar la confluencia de ciertas variables en la organización escolar. Su

Decisiones en evaluación: ambientes virtuales de posgrado, un estudio ex postfacto. Viviana Rocio Rodriguez Aramendiz y Katherina Edith Gallardo Córdova 
naturaleza integradora amerita que los estudiantes realicen procesos que los conducirán finalmente a la Utilización del conocimiento para proponer una solución derivada de la toma de decisiones.

Las actividades 2.8 y 3.6 relacionadas con discutir y socializar, responden a las generalizaciones pues requieren de organización de ideas, detalles, relación entre elementos y brindan información específica. Respondiendo al nivel de procesamiento son actividades que involucran el análisis y la comprensión, la identificación de detalles importantes y la utilización de lo aprendido.

Momento 3: Análisis de los datos en materia de retroalimentaciones emitidas a las actividades ponderadas. Este análisis arrojó que, en su mayoría, se utilizaron rúbricas con criterios que permitían arrojar resultados numéricos con la calificación correspondiente. En algunos casos la retroalimentación iba acompañada con ciertos comentarios emitidos. Frente a los comentarios se enfocaron más en aspectos de forma que de contenido. Se emitieron una serie de indicaciones sobre elementos faltantes en los formatos, faltas de ortografía o errores gramaticales y algunos señalamientos relacionados con la forma de citación y conformación de referencias según el estilo que se determinó como oficial para enviar los trabajos escritos (APA). En cuanto a los comentarios de contenido, aunque se presentaron, estos distaron de contener un carácter orientador para que los estudiantes logren los niveles a los que las actividades apuntaban. Algunos ejemplos de las retroalimentaciones son:

- Eliminar ideas que no concuerdan con el trabajo indicado, datos y gráficas faltantes, porcentajes faltantes

- Eliminar ideas que no concuerdan con el trabajo indicado, datos y gráficas faltantes, porcentajes faltantes, cálculos pendientes y definiciones inadecuadas

- Si se cierra la documentación los indicadores no podrá formar parte de un plan integral de evaluación ya que restringe su existencia al período de vida del Plan 2011

- Lo esperado en el documento eran tablas, gráficos de barras, pays, etc. que representaran en una forma más visual los hallazgos a la comunidad educativa. Si solo se le presentan letras se corre un alto riesgo de perder su atención. Se recomienda trabajar más en esto.

\section{Discusión y conclusiones}

Teniendo en cuenta el análisis de resultados llevado a cabo en dos cursos, modalidad en línea y a distancia de posgrado, se pudo inferir que las actividades diseñadas se estructuraron de tal manera que el sistema de evaluación se construyó sólidamente para que los estudiantes alcancen las meta de aprendizaje. Podría afirmarse que la toma de decisiones por parte de los diseñadores permitió que los

Decisiones en evaluación: ambientes virtuales de posgrado, un estudio ex postfacto. Viviana Rocio Rodriguez Aramendiz y Katherina Edith Gallardo Córdova 
estudiantes alcanzarán los objetivos del curso de forma estructurada y coherente. Con respecto a cada uno de los momentos y teniendo en cuenta el orden en que se analizaron cada uno de los cursos, en el momento 1 , se observa que existe conjunción entre procesos de evaluación individual y grupal, la evaluación se da en dos estilos, formativa y sumativa con cargas manejables, es motivadora dada la diversidad de actividades planteadas donde se genera interacción entre pares (maestro - estudiante) lo cual fortalece la confianza, las competencias y objetivos que direccionaron cada curso fueron claramente establecidos, cada una de ellas en los procesos evaluativos, actividades y el calendario, congruentes unos con otros.

Por su parte en el momento 2, hallazgos sobre alcances de los objetivos/competencias a través de las actividades seleccionadas, la hoja compartida en google docs fue útil en el proceso de recolección y análisis de información, la motivación, la activación de los conocimientos previos, las actividades de aprendizaje, los materiales, las habilidades, los procesos, las actitudes, el entorno de interacción, la orientación, la reflexión y la evaluación, fueron decisivos para determinar el material didáctico o recursos de aprendizaje, analizados cada uno de ellos con los objetivos específicos, están enlazados con cada objetivo general del curso, lo anterior denota que los procesos evaluativos están encaminados a cumplir con las metas establecidas por cada curso, cada uno de ellos relacionado con un dominio del conocimiento y con un nivel de procesamiento, por otro lado, el tiempo y espacio para el desarrollo de las actividades podría extenderse un poco más para hacerlas profundas y mejor elaboradas por parte de los estudiantes.

En el momento 3, análisis de hallazgos en retroalimentaciones dadas a las actividades ponderadas del curso A, se encontró que el grupo es homogéneo y la percepción de la retroalimentación es heterogénea, la retroalimentación dada no provee suficiente información que apoye el aprendizaje de los estudiantes, falto profundidad en los comentarios dados por los profesores, en algunas no había comentarios. Es indispensable trabajar en el fortalecimiento de competencias evaluativas de tipo formativo como lo es la retroalimentación, pues de esa manera el estudiante fortalece sus debilidades y alcance de las metas, además la congruencia de lo que se evalúa con lo solicitando.

Despues de indagar cada uno de los cursos, se concluye que la evaluación es continua, formativa y sumativa, basada en el análisis de procesos. Este tipo de evaluación fortalece el aprendizaje significativo y podría ser una herramienta útil a la hora de evaluar en el aula.

Por su parte, el diseño instruccional de los cursos también soporta los objetivos trazados, pues la tecnología es un agente dinamizador en el proceso educativo.

Teniendo en cuenta que la evaluación implica tomar decisiones para cumplir con los propósitos y metas adecuadas del curso, siempre amerita tomar decisiones para conducir a que arroje información sobre el progreso de los estudiantes. Estas

Decisiones en evaluación: ambientes virtuales de posgrado, un estudio ex postfacto. Viviana Rocio Rodriguez Aramendiz y Katherina Edith Gallardo Córdova 
decisiones pueden irse ajustando, por lo que su uso debe concebirse como un factor que va más allá de la emisión de calificaciones. Es decir, debe trascender y permitirle al estudiante hacer una introspección de sus debilidades y fortalezas para mejorar y ampliar la esencia de la misma, hacia la integración de conocimientos que permita el desarrollo integral del individuo. En este estudio los resultados se aprecian favorables en la toma de decisiones. Se sugiere seguir indagando en el proceso de retroalimentación, tanto en la capacitación que reciben los docentes para su emisión y uso para la mejora continua, como en la utilidad de esta información para los aprendices.

Presentación del artículo: 11 de diciembre de 2018

Fecha de aprobación: 1 de febrero de 2019

Fecha de publicación: 30 de abril de 2019

Rodriguez Aramendiz, V.R. y Gallardo Córdova, K.E. (2019). Decisiones en evaluación: ambientes virtuales de posgrado, un estudio ex post-facto. RED. Revista de Educación a Distancia, 59. DOI:

http://dx.doi.org/10.6018/red/59/06

\section{Financiación}

Esta investigación no ha recibido ninguna subvención específica de los organismos de financiación en los sectores públicos, comerciales o sin fines de lucro.

\section{Referencias}

Alkharusi, H. (2008). Effects of Classroom Assessment Practices on Students'Achievement Goals. Educational Assessment, 13, 243-266. doi:10.1080/10627190802602509

Beauvais, M. (s/f). La evaluación: una cuestión de responsabilidad. Lille, Francia. Obtenido de http://dugidoc.udg.edu/bitstream/handle/10256/3592/Beauvais_es.pdf?sequence $=3$

Benítez Lima, M.G. (2010). El modelo de diseño instruccional Assure aplicado a la educación a distancia. Revista Académica de Investigación Tlatemoani, 2(1), 114. Recuperado de http://www.eumed.net/rev/tlatemoani/01/pdf/6377_mgbl.pdf

Decisiones en evaluación: ambientes virtuales de posgrado, un estudio ex postfacto. Viviana Rocio Rodriguez Aramendiz y Katherina Edith Gallardo Córdova 
Bloom, B.S., Engelhart, M.D., Furst, E.J., Hill, W.H., \& Krathwohl, D.R (1956). Taxonomy of Educational Objectives - The Classification of Educational Goals - Handbook 1: Cognitive Domain. London, WI: Longmans, Green \& Co. Ltd.

Casanova Rodríguez, M. A. (1998). Evaluación: Concepto, tipología y objetivos. La evaluación educativa. Escuela básica, 67-102. Distrito Federal, México.

Consorcio de Educación a Distancia (2014). Seguimiento educación en línea en los Estados. Encuesta de línea de aprendizaje. Disponible en: http://onlinelearningconsortium.org/read/survey-reports-2014/

Córica, J. L. et al. (2010). Fundamentos del diseño de materiales para la educación a distancia. Mendoza, Argentina: Editorial Virtual Argentina.

Creswell, J. y Plano, V. (2007). Designing and conducting mixed methods research. Thousand Oaks, CA, EUA: SAGE.

De la Garza Vizcaya, E.L. (2004). La evaluación educativa. Revista Mexicana de Investigación Educativa, 9(23).807-816. Recuperado de: http://www.oei.es/evaluacioneducativa/evaluacion_educativa_delagarza.p df

De Vizenci, A. y De Angelis, P. (2008). La evaluación de los aprendizajes de los alumnos: Orientaciones para el diseño de instrumentos de evaluación. Revista de Educación y Desarrollo, Abril- junio, (17-22).

Desai, M. S, y Johnson, R. A. (2014). Integrated systems oriented student-centric learning environment. Campus - Wide Information Systems, 31(1), 24-45. doi:http://0-dx.doi.org.millenium.itesm.mx/10.1108/CWIS-01-2013-0002

Dubas M.J, Toledo A.S. (2016). Taking higher order thinking seriously: Using Marzano's taxonomy in the economics classroom. Original Research Article. Pages 12-20

Escudero Escorza, T. (2003). Desde los tests hasta la investigación evaluativa actual: un siglo, el XX, de intenso desarrollo de la evaluación en educación. Revista Electrónica de Investigación y Evaluación Educativa, 9(11). Recuperado de: http://www.uv.es/RELIEVE/v9n1/RELIEVEv9n1_1.htm

Gallardo - Córdova, K.E.; Gil Rendón, M.E. (2012). Utilización de la Nueva Taxonomía para Evaluar el Aprendizaje en Programas de Posgrado en Línea y a Distancia, Articulo. Disponible en: http://rieege.tecvirtual.mx/index.php/rieege/issue/view/5

Gallardo Córdova, K.E. (2013). Evaluación del aprendizaje: retos y mejores prácticas. Monterrey, Nuevo León, México: Editorial Digital del Tecnológico de Monterrey. Disponible en: http://www.amazon.com/Evaluaci\%C3\%B3n-

Decisiones en evaluación: ambientes virtuales de posgrado, un estudio ex postfacto. Viviana Rocio Rodriguez Aramendiz y Katherina Edith Gallardo Córdova 
del-aprendizaje-mejores-pr\%C3\%A1cticas-ebook/dp/B00Q9TBTTM

Gros Salvat, B. (2012). Retos y tendencias sobre el futuro de la investigación acerca del aprendizaje con tecnologías digitales. RED. Revista de Educación a Distancia 3(32). Recuperado de: http://hdl.handle.net/2445/44090

Hernández Sampieri R., Fernández Collado C., Baptista Lucio P. (2006). Metodología de la investigación (4ta ed.). Ciudad de México, México: Editorial Interamericana Mc Graw Hill; p. 220-1.

Instituto Nacional de Evaluación Educativa (INEE) (2008). La evaluación de los aprendizajes en América Latina. Revista Instituto Nacional para la Evaluación de la Educación, (32) D. 5-28. Recuperado de: http://publicaciones.inee.edu.mx/buscadorPub/P1/C/140/P1C140.pdf

Jané, M. (2005). Evaluación del aprendizaje: ¿problema o herramienta?.Revista Informática Educativa UNIANDES-LIDIE, 8(20). 93-98. Recuperado de: http://res.uniandes.edu.co/view.php/451/index.php?id=451

King, L. (2010). Designing and teaching learning goals and objectives: Classroom strategies that work. School Administrator, 67(1), 48.

Law, C y Kaufhold, J. A. (2009). An analysis of the use of critical thinking skills in reading and language arts instruction. Reading Improvement, 46(1), 29-34.

Londoño Giraldo, E.P. (2011). El diseño instruccional en la educación virtual: más allá de la presentación de contenidos. Revista Educación y Desarrollo Social, 5(2). 112-127. Recuperado de: http://www.umng.edu.co/documents/63968/70434/etb_articulo8.pdf

Martínez Rodríguez, A (2009). El diseño instruccional en la educación a distancia. Revista Electrónica de Investigación Educativa, 9(10). 104-119. Recuperado de: http://www.redalyc.org/articulo.oa?id=68812679010

Martínez Rizo, F. (2013). El futuro de la evaluación educativa. Sinéctica Revista

Electrónica de Educación, 40. Recuperado de: http://www.sinectica.iteso.mx/articulo/?id=40_el_futuro_de_la_evaluacion_ educativa

Martínez Uribe, C. H. (2008). La educación a distancia: sus características y necesidad en la educación actual. Revista en educación, XVII (33). 7-27. Recuperado http://webcache.googleusercontent.com/search?q=cache:2kOikqprDTwJ:dia lnet.unirioja.es/descarga/articulo/5057022.pdf $+\& c d=1 \&$ hl=es$419 \& \mathrm{ct}=\mathrm{clnk} \& \mathrm{gl}=\mathrm{cO}$

Marzano R.J. y Kendall, J.S. (2007). The new taxonomy of educational objectives. Thousand Oaks, California, EE.UU. Corwnin Press.

Molenda, (1997). Historical and philosophical foundations of instructional design: A North American view, 1, 41-53.

Decisiones en evaluación: ambientes virtuales de posgrado, un estudio ex postfacto. Viviana Rocio Rodriguez Aramendiz y Katherina Edith Gallardo Córdova 
RED. Revista de Educación a Distancia. Núm. 59, Artíc. X, 30-04-2019

DOI: http://dx.doi.org/10.6018/red/59/06

Shelton, A. R., y Brown, R. S. (2010). Measuring the alignment of high school and community college math assessments. Journal of Applied Research in the Community College, 18(1), 6-15.

Stugis, C., y Patrick, S. (2010). When success is the only option: designing competencybased pathways for next generation learning. Virginia (VA), Estados Unidos: INACOL - International Association of K-12 Online Learning. Obtenido de http://www.competencyworks.org/wpcontent/uploads/2012/04/iNACOL_SuccessOnlyOptn.pdf

Valenzuela González, J. R. y Flores Fahara, M. (2012). Fundamentos de investigación educativa (eBook, Vols. I, II y III). Monterrey, México: Editorial Digital del Tecnológico de Monterrey. 